Frans W. Cornelissen $\cdot$ Hubert Kimmig Mark Schira $\cdot$ Roland M. Rutschmann

R. Paul Maguire $\cdot$ Annelies Broerse

Johan A. Den Boer • Mark W. Greenlee

\title{
Event-related fMRI responses in the human frontal eye fields in a randomized pro- and antisaccade task
}

Received: 12 March 2001 / Accepted: 25 April 2002 / Published online: 30 May 2002

(C) Springer-Verlag 2002

\begin{abstract}
We examined whether the frontal eye fields (FEF) are involved in the suppression of reflexive saccades. Simultaneous recording of horizontal eye movements and functional magnetic resonance imaging enabled us to perform a randomized pro- and antisaccade task and to sort blood oxygenation level dependent (BOLD) time series on the basis of task performance. Saccadic reaction time distributions were comparable across tasks indicating a similar effort in preprocessing of the saccades. Furthermore, we found similar BOLD activation in FEF during both correctly performed proand antisaccades. Frontal eye field activation started prior to target presentation and saccade generation. While we observed only few erroneous antisaccades, these were associated with a decrease in BOLD activity prior to target presentation, and increased BOLD activity after target presentation relative to correctly performed antisaccades. These findings are consistent with a role of the
\end{abstract}

F.W. Cornelissen ( $)$

Laboratory for Experimental Ophthalmology,

School for Behavioral and Cognitive Neurosciences,

University of Groningen, P.O. Box 30.001,

9700 RB Groningen, The Netherlands

e-mail: f.w.cornelissen@med.rug.nl

Tel.: +31-50-3614173, Fax: +31-50-3611709

H. Kimmig · M. Schira

Neurologische Universitätsklinik, Universität Freiburg,

Breisacher Strasse 64, 79106 Freiburg, Germany

R.M. Rutschmann · M.W. Greenlee

Cognitive Neuropsychology, Department of Psychology,

University of Oldenburg, Ammerlaender Heerstrasse 114-118,

Building A6 2-209, 26111 Oldenburg, Germany

R.P. Maguire

Department of Neurology,

School for Behavioral and Cognitive Neurosciences,

University of Groningen, P.O. Box 30.001, 9700 RB Groningen,

The Netherlands

F.W. Cornelissen · A. Broerse · J.A. Den Boer

Department of Biological Psychiatry,

School for Behavioral and Cognitive Neurosciences,

University of Groningen, P.O. Box 30.001, 9700 RB Groningen,

The Netherlands
FEF in the suppression of reflexive saccades. The increase in activity after target presentation for antisaccade errors can only be indirectly linked to such a role but may also reflect activity related to the generation of a correction saccade. Frontal eye field BOLD activity may further represent general arousal, preparatory set, shortterm memory, or salience-map related activity.

Keywords Event-related fMRI · Prosaccades . Antisaccades · Frontal eye fields

\section{Introduction}

Antisaccades are voluntary saccades made toward the mirror position of a visual object, i.e., a "virtual" target defined purely by instruction (Hallet 1978; Hallet and Adams 1980). To correctly perform an antisaccade, a reflexive (pro-) saccade toward the suddenly appearing visual stimulus needs to be suppressed. Frequently, errors occur that indicate that the subject was unable to do so. In this study, we focused on the role of the frontal eye fields (FEF) in this suppression of reflexive saccades while subjects make antisaccades. In humans, the FEF are located in the vicinity of the precentral sulcus in Brodmann area 6 (Paus et al. 1996). The FEF are part of a cortical network that controls saccadic eye movements (Gaymard et al. 1998) and have a specific role in the generation of voluntary saccades, such as antisaccades (Fischer and Boch 1991).

A number of recent human neuroimaging studies (O'Driscoll et al. 1995; Sweeney et al. 1996; Doricchi et al. 1997; Connolly et al. 2000; Kimmig et al. 2001) reported more activation of the FEF associated with antisaccades relative to prosaccades. One study (Muri et al. 1998) did not observe such a difference. The additional activity in the FEF during antisaccades supports the involvement of this area in the generation of voluntary saccades. The studies cited above contrasted activation when subjects performed the pro- and antisaccade trials blockwise, such that no uncertainty with respect to trial 
type was present. The differences in activation must thus be related to the additional processing (i.e., response suppression, trajectory programming) required for the correct execution of antisaccades. Additional, albeit indirect, evidence for a role of the FEF in the suppression of reflexive saccades comes from an event-related functional magnetic resonance imaging (fMRI) study of overt and covert shifts of attention (Perry and Zeki 2000). In this study, the FEF were more active during covert shifts of attention that required suppression of saccades than during overt shifts that required (pro-) saccade generation. In monkeys, Burman and Bruce (1997) found that in nearly half of the sites tested, low threshold electrical stimulation of the FEF prior to visual, memory-guided, and antisaccades led to an increase in saccadic latency, again supporting a role of the FEF in the suppression of saccades.

An indication against a role of the FEF in reflexive saccade suppression comes from a lesion report by Gaymard et al. (1998, 1999). A subject with a small unilateral lesion restricted to the FEF showed a normal error rate in the antisaccade task. Gaymard et al. interpreted this as indicating that the FEF have no role in reflexive saccade suppression. In a monkey study, Everling and Munoz (2000) found decreased activity in certain FEF cells (those projecting directly to the superior colliculus) during and prior to antisaccades relative to prosaccades. Less activity on the antisaccade task, during which suppression should be most pronounced, is an indication that these particular FEF cells, at least, had no role in preventing reflexive eye movements. However, the decrease in activity could well be the result of other (FEF) neurons inhibiting this particular subset of cells.

A direct comparison of these single cell, imaging, and lesion studies is hampered by both methodological differences as well as the difficulty to directly compare neuroimaging and single cell results. The results so far are not unequivocal and it thus remains unclear whether the FEF have a role in the suppression of spontaneous and reflexive saccades. Without expecting to immediately resolve this issue, we decided to employ an approach frequently used in monkey physiology (see, for example, Everling and Munoz 2000) in a human event-related fMRI experiment in order to gain additional insight in the role of the FEF. The approach is to randomize the presentation of pro- and antisaccades on a trial-by-trial basis. Only briefly before the presentation of the target the expected saccade type is indicated, making the task unpredictable. Subjects must therefore prepare themselves in a similar way for each type of task, eliminating strategy-related differences from influencing the results. Indeed, when pro- and antisaccades are randomized in such a way, their latencies tend to become quite similar (Weber 1995). This similarity supports the idea that randomizing pro- and antisaccade trials renders the prosaccades voluntary, rather than reflexive, such as in a block paradigm. Thus, at a behavioral level at least, pro- and antisaccades become much more comparable under the condition of randomized presentation.

\section{Materials and methods}

Our methods for visual stimulation, recording and analysis of eyemovement data, and acquisition of fMRI data have been described elsewhere (Kimmig et al. 1999, 2001). We here only describe details that were specific to the current study.

Stimulus presentation during the randomized pro- and antisaccade task was as follows. A blue fixation spot was positioned in the center of the display at the beginning of each trial. Two different conditions were performed, a prosaccade and an antisaccade task. A change in color of the fixation spot, $500 \mathrm{~ms}$ prior to target onset indicated the type of saccade to make. A change to green indicated the subject had to make a prosaccade, whereas a change to red indicated an antisaccade trial. During the saccadic eye-movement task, the fixation spot was extinguished $200 \mathrm{~ms}$ prior to the onset of the peripheral $\left(10^{\circ}\right.$ to the left or right $)$ target. The subject was asked to make a saccade as quickly as possible to the target location. After $1.5 \mathrm{~s}$ the target disappeared and the central fixation spot appeared again in the center of the display and subjects relocated their gaze to the fixation spot.

Eye-movement data were used to categorize pro- and antisaccade trials into correct and incorrectly performed trials and lapses, as well as used to determine saccade latency (difference between onset of the target and start of the saccade). The fMRI data for lapses and saccades with a latency shorter than $80 \mathrm{~ms}$ (classified as anticipatory) were discarded, but since this occurred rarely (less than $5 \%$ of trials) no further analysis on these data was conducted.

\section{Event-related fMRI data acquisition and analysis}

Functional imaging was performed with $\mathrm{T} 2 *$-weighted gradient recalled echoplanar imaging. (The technical data for the functional measurements were TE $66 \mathrm{~ms}$, TR $1.5 \mathrm{~s}$, flip angle $90^{\circ}$, field of view $256 \mathrm{~mm}$, six slices, matrix $128 \times 128$, voxel size of $2 \times$ $2 \times 4 \mathrm{~mm}$.) During the stimulation protocol 128 volumes were acquired over a $3.2-$ min period. In this period, 10 saccade trials were presented to the subject (five pro- and five antisaccades randomly interleaved). Although this "sparse" presentation allowed us to present only relatively few trials in a run, it does allow us to completely follow the rise and fall of the blood oxygenation level dependent (BOLD) signal. Subjects performed four to six runs each, such that 40-60 saccade trials were collected for each subject.

Left and right hemispheric ROIs in the human FEF were determined both on the basis of the anatomical location and by requiring that the time course of the BOLD activation was correlated with the presentation of saccade targets (irrespective of the type of saccade or performance). The ROI was $6 \times 6$ voxels centered on the maximum of activation. Time courses in the thus defined ROIs were extracted from the data of each run for both left and right hemispheres.

Of each run, the first four volumes were discarded to remove saturation onset effects. The raw time course data were first corrected for slow linear changes in mean signal intensity over the entire time series. The data were converted to percent signal change by subtracting and dividing by the mean signal intensity of the time series during each trial. The BOLD responses associated with each type of trial were then separately averaged. The fMRI response amplitudes were computed from the time series as described by Ress et al. (2000) and were used to scale the responses of each subject. To have a fair number of erroneous antisaccades contributing to the results we only present data combined over subjects. The presence of statistically significant differences in the fMRI time series and latency data was verified using ANOVA with (when applicable) subject, hemisphere, saccade type and error, and time from stimulus onset as independent variables. Post hoc analysis was performed using Scheffe's $F$ procedure.

\section{Subjects}

After giving their informed consent, three volunteers participated in the study. The subjects' age ranged from 24 to 36 years. All subjects were males, one of whom was author F.W.C. 

of saccadic reaction times for prosaccades (A) and antisaccades (B). Bin width $20 \mathrm{~ms}$
Fig. 1 Frequency distributions
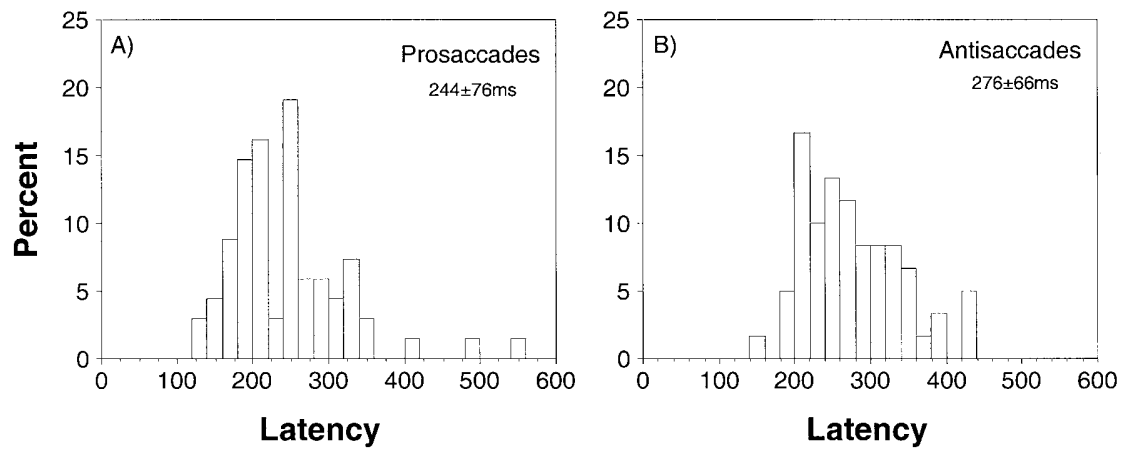

Fig. 2 A Average blood oxygenation level dependent (BOLD) responses of three subjects for correct pro- and correct and erroneous antisaccades in the frontal eye fields (FEF). B Average BOLD responses of three subjects averaged over correct pro- and antisaccades in the FEF and area V1 (primary visual cortex). The saccade target appeared at $\mathrm{t}=0 \mathrm{~s}$ (bars show $\mathrm{SEM})$
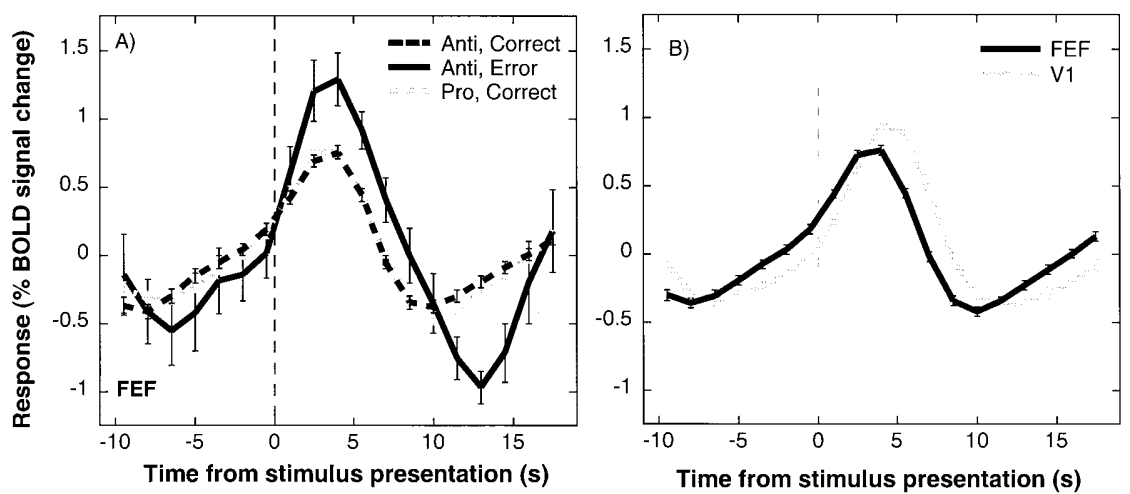

Table 1 Percentage of correct and incorrect anti- and prosaccades for each subject

\begin{tabular}{llll}
\hline Subject & \multicolumn{2}{l}{ Antisaccades } & Prosaccades \\
\cline { 2 - 3 } & Correct & Error & Correct \\
\hline F.C. & $49 \%$ & $2 \%$ & $49 \%$ \\
J.J. & $32 \%$ & $13 \%$ & $55 \%$ \\
R.E. & $48 \%$ & $2 \%$ & $50 \%$ \\
\hline
\end{tabular}

\section{Results}

Data were acquired for three subjects [mean Talairach coordinates (Talairach and Tournoux 1988) for the FEF averaged over subjects and hemispheres: $x=40.6( \pm 4.9)$, $y=-8.5( \pm 3.8), z=55.4( \pm 4.5)$; number in brackets is standard deviation]. Table 1 lists the percentage of correct and incorrect anti- and prosaccades for each subject. Figure 1 shows that the latency distributions for pro- and antisaccades were fairly comparable (median, skewness, and variance of $239,1.5,5,586$ and $266,0.6,4,333$, respectively). There were significant differences in latency associated with the different response and saccade types $[F(2,125)=4.6 ; P<0.05]$. Post hoc analysis showed that the small difference of $32 \mathrm{~ms}$ between correct pro- and antisaccades (mean latencies of 244 and $276 \mathrm{~ms}$, respectively) reached significance $(P<0.01)$. Latencies for correct and incorrect antisaccades differed by $95 \mathrm{~ms}$ (mean latencies 276 and $181 \mathrm{~ms}$, respectively), which was highly significant $(P<0.001)$.
Figure 2A shows the BOLD responses in the FEF, averaged over left and right hemispheres. Note that the BOLD responses for correctly performed pro- and antisaccades are highly comparable. In contrast, there was a clear difference in the BOLD response of correct and incorrect antisaccades. Prior to target appearance BOLD activity for erroneous antisaccades was slightly lower than for correct ones. For up to $9 \mathrm{~s}$ after target appearance, however, BOLD activity for erroneous antisaccades was higher than for correct ones. About $10 \mathrm{~s}$ after target appearance an undershoot was measured. There were significant differences in BOLD response over both the $7 \mathrm{~s}$ prior to target appearance as well as the first $9 \mathrm{~s}$ after target appearance associated with the different response and saccade types $[F(2,1205)=4.9 ; P<0.01$ and $F(2,1446)=7.7 ; P<0.001$, respectively]. For both time spans, post hoc analysis showed that the difference between pro- and antisaccades was not significant, whereas the difference between correct and erroneous antisaccades was significant $(P<0.05$ and $P<0.001$ for time spans prior to and after target presentation, respectively). All antisaccade errors were followed by a correction saccade.

Figure $2 \mathrm{~B}$ shows the BOLD response averaged over all correct saccades in area V1 (primary visual cortex) and in FEF. The BOLD activity in FEF starts to rise well before target presentation and does so in a much more pronounced way than in $\mathrm{V} 1$. 


\section{Discussion}

We find that when saccades to both real (prosaccades) and virtual objects (antisaccades) are presented in a randomized manner, saccade latency, distributions, and BOLD responses in FEF are similar. The mean difference in latency for pro- and antisaccades in our randomized paradigm of $32 \mathrm{~ms}$ is comparable to the 28 and $27 \mathrm{~ms}$ difference reported by Weber (1995) for a cue lead time of 0 and $300 \mathrm{~ms}$, respectively (our cue lead time was $500 \mathrm{~ms})$. The difference is much smaller than in a regular block design [for example, Weber (1995) reported a difference of $72 \mathrm{~ms}$ and Kimmig et al. (2001) found a difference of $50 \mathrm{~ms}$. Peaks in the frequency distribution of reaction times indicate different processing stages of saccades (Fischer and Weber 1998). The distributions for pro- and antisaccades were comparable. We take this as an indication for similar preprocessing of pro- and antisaccades in our paradigm. The similarity in latency also supports the idea that randomizing the trial order for pro- and antisaccades renders the prosaccades voluntary, rather than reflexive, the latter of which occurs in a non-random block paradigm. In such a paradigm, the differential activity for pro- and antisaccades has been taken to indicate FEF involvement in suppression. In the randomized paradigm, suppression is required prior to both pro- and antisaccades. The similarity in BOLD response for both saccade types can thus be taken as an indirect indication for a role of the FEF in the suppression of reflexive saccades.

The BOLD response in the FEF started to rise well before the appearance of the target and before the saccade was generated indicating the presence of presaccadic neural activity. A direct comparison between areas is somewhat complicated by potential influences of differences in vascularization. We nevertheless think that such a comparison may provide information about the functional origin of BOLD signals. Also in V1 the BOLD signal rose slightly prior to target appearance, yet in FEF this was much more pronounced and occurred at an earlier moment in time. This suggests that this early rise is not just a reflection of a general increase in arousal or attention but rather is related to specific neural processing taking place in FEF. The early rise in activity is rather gradual in comparison to the usually reported event-related BOLD response. Since these neural control processes are internally triggered the saccadic event can only serve as a relative time marker, similar to the "Bereitschaftspotential" in the electric and magnetic encephalography literature (Kristeva-Feige et al. 1997). As the actual time of onset is uncertain, the resulting BOLD response is temporally smoothed.

Only very few erroneous saccades were included in this study. Nevertheless, we will try to interpret the associated BOLD response. The early rise in FEF BOLD signal with correct pro- and antisaccades could be reflecting neural activity related to inhibitory processing. To the extent that errors are indeed due to a failure to suppress reflexive saccades to the visual target, the slight decrease in activity associated with erroneous antisaccades prior to target appearance is consistent with this idea. After target appearance, the FEF BOLD response for erroneous antisaccades rose above that for correct ones. This increase in BOLD activity appears inconsistent with inhibitory processing. However, BOLD activity increases almost linearly with the frequency with which saccades are elicited (Kimmig et al. 2001). As all erroneous saccades were corrected, a parsimonious explanation is that the late increase reflects additional neural activity related to the generation of this correction saccade.

In conclusion, the similarity in pro- and antisaccaderelated responses as well as the early increase in activity are consistent with a role for the FEF in inhibitory activity. Also the decrease in activity prior to antisaccade errors is consistent with such a role. The increase, rather than a decrease, in activity after antisaccade errors can only be reconciled in an indirect way with such a role, but may also be reflecting activity related to the generation of a correction saccade. Presaccadic BOLD activity in the FEF is probably not exclusively related to inhibition. It could also be reflecting preparatory set-related activity (Everling and Munoz 2000), the storage of parameters for a forthcoming saccade (Gaymard et al. 1998, 1999), or the build up of a salience map that marks stimulus locations for their relevance (Thompson and Bichot 1999).

Acknowledgements M.W.G. was supported by Deutsche Forschungsgemeinschaft (SFB 517, C9). Authors F.W.C., A.B., R.P.M., and J.A.B. performed this study in the context of the Groningen University Neuroimaging Program (GNIP).

\section{References}

Burman DD, Bruce CJ (1997) Suppression of task-related saccades by electrical stimulation in the primate's frontal eye field. J Neurophysiol 77:2252-2267

Connolly JD, Goodale MA, Desouza JF, Menon RS, Vilis T (2000) A comparison of frontoparietal fMRI activation during anti-saccades and anti-pointing. J Neurophysiol 84:16451655

Doricchi F, Perani D, Incoccia C, Grassi F, Cappa SF, Bettinardi V, Galati G, Pizzamiglio L, Fazio F (1997) Neural control of fastregular saccades and antisaccades: an investigation using positron emission tomography. Exp Brain Res 116:50-62

Everling S, Munoz DP (2000) Neuronal correlates for preparatory set associated with pro-saccades and anti-saccades in the primate frontal eye field. J Neurosci 20:387-400

Fischer B, Boch R (1991) Cerebral cortex. In: Cronly-Dillon (ed) Vision and visual dysfunction, vol 8. Eye-movements. MacMillan Press, London

Fischer B, Weber H (1998) Effects of pre-cues on voluntary and reflexive saccade generation. I. Anti-cues for pro-saccades. Exp Brain Res 120:403-416

Gaymard B, Ploner CJ, Rivaud S, Vermersch AI, PierrotDeseilligny C (1998) Cortical control of saccades. Exp Brain Res 123:159-163

Gaymard B, Ploner CJ, Rivaud-Pechoux S, Pierrot-Deseilligny C (1999) The frontal eye field is involved in spatial short-term memory but not in reflexive saccade inhibition. Exp Brain Res 129:288-301

Hallet PE (1978) Primary and secondary saccades to goals defined by instructions. Vision Res 18:1279-1296 
Hallet PE, Adams BD (1980) The predictability of saccade latency in a novel voluntary oculomotor task. Vision Res 20:329-339

Kimmig H, Greenlee MW, Huethe F, Mergner T (1999) MR-Eyetracker: a new method for eye movement recording in functional magnetic resonance imaging. Exp Brain Res 126:443-449

Kimmig H, Greenlee MW, Gondan M, Schira M, Kassubek J, Mergner T (2001) Relationship between saccadic eye movements and cortical activity as measured by fMRI: quantitative and qualitative aspects. Exp Brain Res 141:184-194

Kristeva-Feige R, Rossi S, Feige B, Mergner T, Lucking CH, Rossini PM (1997) The Bereitschaftspotential paradigm in investigating voluntary movement organization in humans using magnetoencephalography (MEG). Brain Res Brain Res Protoc $1: 13-22$

Muri RM, Heid O, Nirkko AC, Ozdoba C, Felblinger J, Schroth G, Hess CW (1998) Functional organisation of saccades and antisaccades in the frontal lobe in humans: a study with echo planar functional magnetic resonance imaging. J Neurol Neurosurg Psychiatry 65:374-377

O'Driscoll GA, Alpert NM, Matthysse SW, Levy DL, Rauch SL, Holzman PS (1995) Functional neuroanatomy of antisaccade eye movements investigated with positron emission tomography. Proc Natl Acad Sci USA 92:925-929

Paus T (1996) Location and function of the human frontal eyefield: a selective review. Neuropsychologia 34:475-483

Perry RJ, Zeki S (2000) The neurology of saccades and covert shifts in spatial attention: an event-related fMRI study. Brain 123:2273-2288

Ress D, Backus BT, Heeger DJ (2000) Activity in primary visual cortex predicts performance in a visual detection task. Nat Neurosci 3:940-945

Sweeney JA, Mintun MA, Kwee S, Wiseman MB, Brown DL, Rosenberg DR, Carl JR (1996) Positron emission tomography study of voluntary saccadic eye movements and spatial working memory. J Neurophysiol 75:454-468

Talairach J, Tournoux P (1988) Co-planar stereotaxic atlas of the human brain. Thieme, Stuttgart

Thompson KG, Bichot NP (1999) Frontal eye field: a cortical salience map. Behav Brain Sci 22:699-700

Weber H (1995) Presaccadic processes in the generation of proand antisaccades in human subjects: a reaction time study. Perception $24: 1265-1280$ 